\title{
Virtual sensor fusion for high precision control
}

\author{
Mohit Verma ${ }^{\mathrm{a}, *}$, Thomas Dehaeze ${ }^{\mathrm{c}}$, Guoying Zhao ${ }^{\mathrm{b}}$, Jennifer Watchi ${ }^{\mathrm{b}}$, \\ Christophe Collette ${ }^{\mathrm{b}, \mathrm{c}}$ \\ ${ }^{a}$ CSIR - Structural Engineering Research Centre, Taramani, Chennai 600 113, Tamil Nadu, India \\ ${ }^{\mathrm{b}}$ Université Libre de Bruxelles, Precision Mechatronics Laboratory, BEAMS Department, 50, F.D. Roosevelt av., 1050 Brussels, Belgium \\ ${ }^{\mathrm{c}}$ University of Liège, Department of Aerospace and Mechanical Engineering, Allée de la Découverte 9, 4000 Liège, Belgium
}

\section{A R T I C L E I N F O}

\section{Article history:}

Received 2 February 2020

Received in revised form 18 August 2020

Accepted 22 August 2020

\section{Keywords:}

Sensor fusion

Precision

Vibration isolation

Active control

Virtual sensor

\begin{abstract}
A B S T R A C T
High performance control requires high loop gain and large control bandwidth. However, the spurious resonances at the higher frequencies can limit the performance of such type of systems. This drawback can be overcome by using sensor fusion technique. In sensor fusion, two or more sensors are combined in synergy such that good performance is achieved at lower frequencies while ensuring robustness of the system at higher frequencies. This paper presents a new technique, termed as "virtual sensor fusion", in which only one of the sensors is physically installed on the system while the other sensor is simulated virtually. The virtual sensor is selected based on desired high frequency response. The effectiveness of the proposed technique is demonstrated numerically for a case of active seismic isolation. A robustness analysis of virtual sensor fusion is also carried out in order to study its stability in the presence of spurious resonances. Finally, the technique is experimentally verified on active isolation of pendulum system from ground motion. The results obtained demonstrate good isolation performance at lower frequencies and robustness to plant uncertainties (spurious resonances) at higher frequencies. This technique can be effectively used for high precision control of sensitive instruments.
\end{abstract}

(c) 2020 Elsevier Ltd. All rights reserved.

\section{Introduction}

Precision control applications can be broadly classified into two major categories [1] - disturbance rejection and pointing control. Disturbance rejection involves the isolation of an equipment from a source of vibration or ground motion [2-4]. Seismic isolation of large physics instruments like Laser Interferometer Gravitational-Wave Observatory (LIGO) is one such example [5-7]. Pointing control usually involves tracking or alignment of the reference to a receptor. Tracking the position of a star and telecommunication are some examples where pointing control is required. Also, there are certain applications which require both active isolation and pointing control. For example, microvibration control for high pointing accuracy in space systems [8-10].

In precision control, the gain of the controller is usually set to a very large value in order to achieve the desired performance. This often destabilize the system due to amplification of the internal modes. This can be avoided by collocating sensor and actuator pair. Collocation refers to as placing sensor and actuator at the same location on the structure. The collocated system has alternating poles and zeros, which guarantees the stability of the system [11,12]. However, it is not always possible to collocate sensor and actuator pair due to size and space limitations. Even if they are collocated, there

\footnotetext{
* Corresponding author.

E-mail address: mohitverma@serc.res.in (M. Verma).
} 
are chances that the high frequency flexible modes of the structure might get excited [13]. This limits the bandwidth and the performance of the precision control system. A possible solution to address this issue consists of adding a notch filter at the location of the internal resonances [14,6]. Another way is to passively damp the spurious resonance using tuned mass dampers [15-17] or dynamic vibration absorbers [18]. A major drawback of these solutions is that they require a good knowledge of the plant and the location of the spurious resonance. Also, the variations in the properties of the plant with time will shift the location of the spurious resonance and thus, will render the solution inefficient.

As precision control has to operate in a limited frequency range, one of the effective ways is to use sensor fusion [19]. In sensor fusion two different sensor are used - one sensor is used to achieve good performance in the region of interest while the other imparts stability to the system beyond the region of interest [20]. Sensor fusion also has the ability to mitigate DC drifts [21]. Some of the sensor often have unwanted DC offset which can cause drift in the system. The sensor signal when passed through a high-pass filter is able to remove the DC offset. Since in sensor fusion one of the sensor signals is passed through the high-pass filter, it is able to compensate for the DC drifts. The use of sensor fusion for active vibration isolation was explored in [22] and this paper further develops those ideas. This paper presents virtual sensor fusion in which only one sensor is mounted on the structure while the other sensor is simulated virtually using a digital filter. The control architecture forms an inner loop with the virtual sensor and an outer loop with the real sensor.

Various model based control methods also use a similar kind of control architecture. For example, internal model control [23-25] and disturbance observer based control [26-30] use a model of the plant in the inner loop. The difference between the responses of the real plant and the model (due to uncertainties and/or unmodeled plant dynamics) is then used to drive the controller with the objective of disturbance rejection. The control architecture of virtual sensor fusion is motivated from model based control methods. However, the key difference here is the role of the inner loop. In model based methods, inner loop is added with the aim of achieving good disturbance rejection. However in virtual sensor fusion, the inner loop is added to impart stability to the system in the high frequency region. Also, the inner loop does not necessarily include the model of the plant in virtual sensor fusion. It can be any stable transfer function with the desired high frequency response, provided that it has the same gain and slope as the real plant in the vicinity of the merging frequency (cutoff frequency set for the filters used for fusion), for ensuring a smooth transition between the real plant and the virtual plant. Virtual sensor fusion results in a higher order controller due to the inclusion of the virtual sensor dynamics. Virtual sensor fusion is fundamentally different from higher order control schemes like pole-placement [31], model-based control [32] and optimal control [33]. In virtual sensor fusion, the plant is made to resemble virtual sensor at higher frequencies where the dynamics of the plant are not well known. On the other hand, higher order control schemes require good knowledge of the plant at higher frequencies. Pole-placement and model based methods basically use model of the plant for the controller design and do not explicitly consider uncertainties (uncertainties are usually addressed by designing controller with sufficient gain and phase margins). This implies that the measurements from the actual plant would still be having the spurious resonances which may destabilize the system in some cases. Optimal control can include parametric and norm-bounded uncertainties in the control design. However, the uncertainties are modeled based on the knowledge of the plant. The main aim of the virtual sensor fusion is to bypass the region where the dynamics of the plant are not well known to ensure the stability of the system. The other way to look at this is that we are adding inaccuracies in the measurements from the plant to enhance the stability of the system. This implies that there is a trade off between performance and stability in virtual sensor fusion. It should also be noted that virtual sensor fusion can encompass state-of-the-art control methods for designing of the controller. In this paper, we demonstrate that virtual sensor fusion provides good isolation performance in low frequency region while ensuring stability of the system to high frequency spurious resonances for a single-input single-output (SISO) system. Virtual sensor fusion can be generalized to a multi-input multi-output (MIMO) system but this would require the design of complementary filters for the fusion. H-infinity synthesis can be employed for the design of complementary filters [34].

The organization of this paper is as follows. The comparison of classical and virtual sensor fusion in presented in Section 2. A general framework for virtual sensor fusion is described in Section 3. It demonstrates how the control input is decoupled from the high frequency spurious resonances of the plant. Virtual sensor fusion is then applied to active seismic vibration isolation system in Section 4. This is followed by robust stability analysis of the same system studied in Section 5. Finally, the experiments carried out towards the validation of virtual sensor fusion on a pendulum system are presented in Section 6 followed by concluding remarks in Section 7.

\section{Classical vs. virtual sensor fusion}

Classical sensor fusion involves the fusion of the measurements from different sensors [5]. The sensors are physically installed on the structure and complementary filters are used for the fusion. An example of classical sensor fusion on a single degree of freedom system is shown in Fig. 1. In the figure, $m$ is the mass, $k$ is the stiffness, $c$ is the damping, $x$ is the displacement, $w$ is the ground motion, $u$ is the control input, $H_{H}$ and $H_{L}$ are the high- and low-pass filters, $K$ is the controller, $G_{v}$ is the virtual sensor and $y, y_{1}, y_{2}$ corresponds to the sensed signals. The physical sensors installed on the structure corresponds to the inertial $\left(y_{1}\right)$ and the force $\left(y_{2}\right)$ sensors. The complementary filters, $H_{H}$ and $H_{L}$, are used for the fusion. The fused measurement signal is then sent to the controller, $K$, for the evaluation of the control input, $u$, to the actuator. In case of virtual sensor fusion, only one sensor is physically installed on the structure (inertial sensor in current example). The other sensor is virtual $\left(G_{v}\right)$. The advantages of virtual sensor fusion over classical sensor fusion are as follows. 


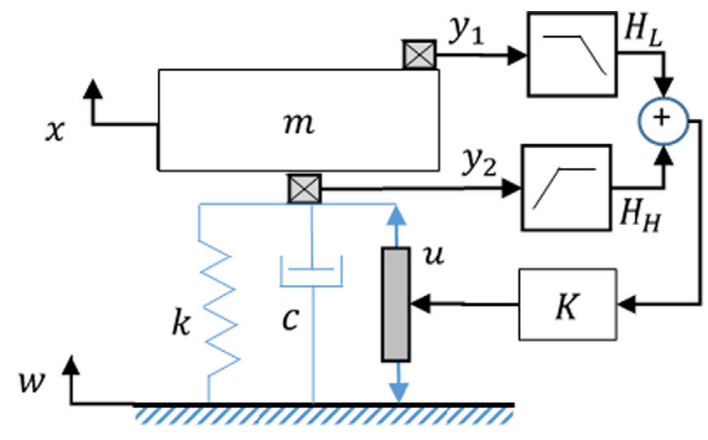

Classical sensor fusion

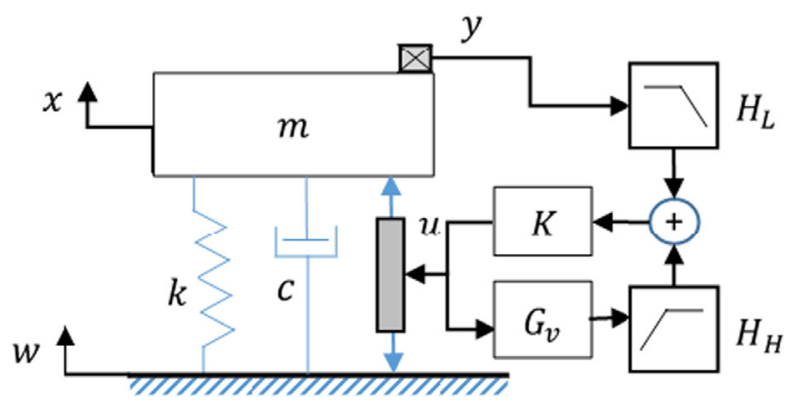

Virtual sensor fusion

Fig. 1. Comparison of classical sensor fusion with the virtual sensor fusion.

(i) It requires installation of only one physical sensor and hence, is more economical.

(ii) It allows to bypass the high frequency spurious resonances of the system.

(iii) In classical sensor fusion, the dynamics of the sensor which is passed through a high-pass filter are fixed which depends upon the physical design of the sensor. However, in case of virtual sensor fusion, the dynamics of the virtual sensor can be tuned as per the desired high-frequency response.

(iv) One might also encounter a case where large array of sensors cannot be installed on the structure due to space limitations. Virtual sensor fusion is deemed useful for such cases.

(v) Installation of large and heavy sensor can modify the physical properties of the primary structure. Virtual sensor fusion does not modify the properties of the primary structure as the sensor dynamics are simulated virtually.

A major disadvantage of using virtual sensor fusion is that it requires additional check for stability. One need to ensure the stability of the inner loop in addition to overall system (see Section 3 for details). Other disadvantages include reduced accuracy and observability at higher frequencies.

\section{Control architecture}

A generalized framework for virtual sensor fusion is shown in Fig. 2 . In the figure, $K$ is the controller to be designed, $G_{u}$ is the plant, $G_{v}$ is the virtual sensor, $H_{L}$ is the low pass filter, $H_{H}$ is the high pass filter, $r$ is the reference signal (equal to zero in case of isolation system), $u$ is the control input to the plant, $y$ is the output to be regulated, $d$ is the disturbance acting at the output of the plant and $n$ is the measurement noise. A real sensor (inertial sensor is used in the present study) is used at low frequencies to provide good performance while a virtual sensor is used at high frequencies to ensure stability of the closed loop system. The virtual sensor is the output of a virtual plant, $G_{v}$, designed to dominate the control command at high frequency. It does not need to mimic a physical quantity of the real plant. The sensors are augmented with filters such that the

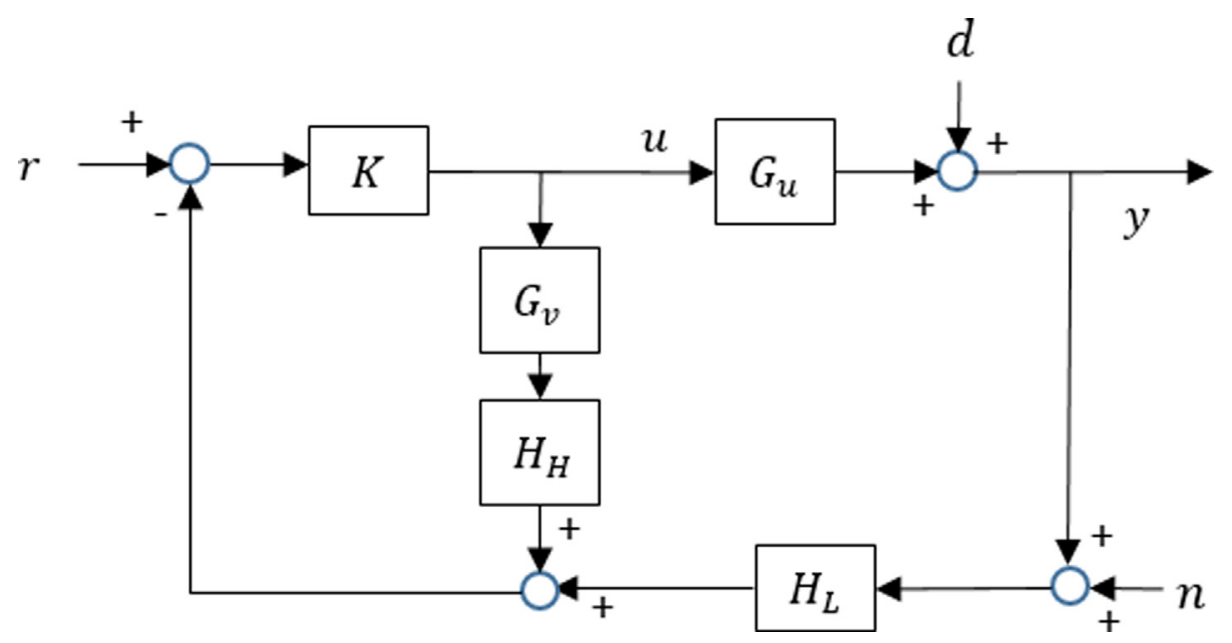

Fig. 2. Generalized framework for virtual sensor fusion. 


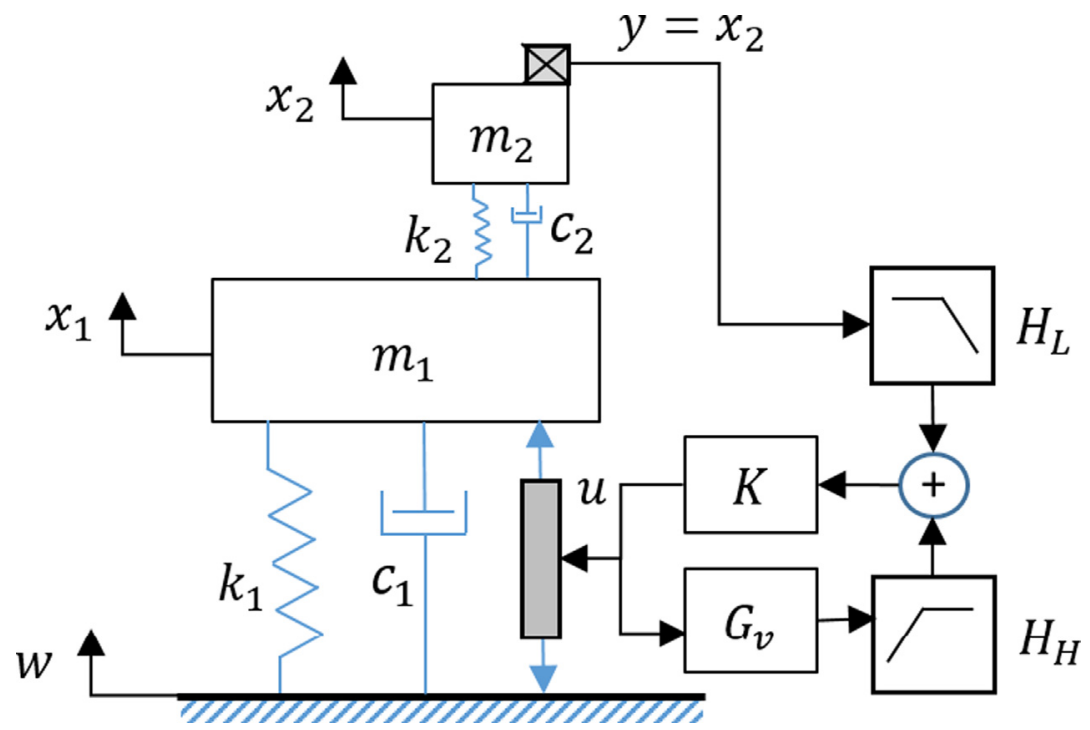

Fig. 3. Virtual sensor fusion applied to the active vibration isolation.

control input is dominated by the real sensor at lower frequencies and by virtual sensor at higher frequencies. It is assumed that the order of the filters $H_{L}$ and $H_{H}$ are chosen in such a way that $G_{u} H_{L} \gg G_{v} H_{H}$ at low frequencies while $G_{v} H_{H} \gg G_{u} H_{L}$ at high frequencies even in the presence of lightly damped modes. A new sensor is constructed by fusing the signals from the inertial and virtual sensors. Based on the block diagram, the output to be regulated is equal to

$$
y=\frac{1+K G_{v} H_{H}}{1+L} d+\frac{K G_{u}}{1+L} r-\frac{K G_{u} H_{L}}{1+L} n
$$

where $L=K\left(G_{v} H_{H}+G_{u} H_{L}\right)$ is the loop gain. A system is said to have good tracking performance when the output of the system, $y$, tracks the reference input signal, $r$. A system is also said to have good disturbance rejection when the output of the system, $y$, is unaffected by the disturbance, $d$, acting on the system. It is observed that good tracking performance or disturbance rejection can be obtained if a large value of the gain is chosen for the controller $K$ at low frequency. In classical control architecture, increasing the gain might lead to gain crossover frequency near the spurious resonances of the system. This results in the phase drop of the open-loop gain, $L$, beyond $\pm \pi$ which renders the closed loop system unstable. However, such problems are bypassed if we use virtual sensor fusion. This can be demonstrated by observing the control input to the plant. The control input, $u$, is given by

$$
u=-\frac{K H_{L}}{1+L} d+\frac{K}{1+L} r-\frac{K H_{L}}{1+L} n
$$

The control input at the low frequencies can be approximated as

$$
u=-\frac{K}{1+K G_{u}} d+\frac{K}{1+K G_{u}} r-\frac{K}{1+K G_{u}} n
$$

It can be seen that at low frequency, the control input solely depends on the measurements from the sensor mounted on the actual structure (inertial control). Similarly, it can be shown that the control input at higher frequencies (obtained by substituting $H_{L} \approx 0$ and $L \approx K G_{v} H_{H}$ in Eq. (2)) can be written as

$$
u=\frac{K}{1+K G_{v} H_{H}} r
$$

The control input at high frequency depends only on the virtual sensor. Thus, the instability that might occur due to the spurious resonances of the system at the higher frequencies is avoided as they do not appear in the feedback loop. The stability of the closed loop system is usually investigated by observing the open loop gain response of the system. When the controller is designed based on the nominal model of the plant, the open loop gain of the system can be written as

$$
L=K G_{u}
$$

The robust stability of the system is guaranteed when the phase of the open loop is away from $\pm \pi$ at the gain crossover frequency. The spurious resonances (which are not accounted in the nominal model) results in a phase drop. There can be a case where increasing the gain results in phase to be $\pm \pi$ at the gain cross over frequency. Even with a lowpass filter there will be phase drop due to the spurious resonances and instabilities may be encountered when the spurious resonances are 
lightly damped. In addition, the introduction of lowpass filters also undermine the control effectiveness in the frequency range of interest as it essentially reduces the control bandwidth. In case of virtual sensor fusion, the open loop gain of the system is given by

$$
L=K\left(G_{v} H_{H}+G_{u} H_{L}\right)
$$

It can be seen that the open loop gain at higher frequency is independent of the plant dynamics and hence, the spurious resonances are by-passed. This avoids any potential phase drop in the open loop frequency response.

The stability of closed loop system is not sufficient to ensure the stability of virtual sensor fusion because of the innerloop. The control signal $u$ and $y$ are related by the following transfer function

$$
G_{\text {inner }}=\frac{K H_{L}}{1+K G_{v} H_{H}}
$$

The stability of the above transfer function should also be checked in addition to the overall stability of the closed loop system. Actually, in the frequency range below the merging, an additional constrain might be imposed on $\mathrm{K}$ for ensuring the stability of the internal loop, because the two loops do not have the same gain at low frequency. However, if the stability of the full system (Eq. (1)) is conditional, the stability of the internal loop is automatically guaranteed. Any classical method can be adopted for designing $K$. The open loop gain is dependent on the controller. Hence, the performance of the system will vary with the choice of the controller.

\section{An example of active vibration isolation using virtual sensor fusion}

In this section, we demonstrate the application of virtual sensor fusion for vibration isolation. The payload to be isolated is constituted of two masses $m_{1}$ and $m_{2}$, linked by a stiffness $k_{2}$ and a dashpot $c_{2}$ in order to represent the flexibility of the payload (Fig. 3). The bottom spring-mass-dashpot system represents the system to be isolated while the top represents sensor dynamics. The input of the controller is the inertial motion of the top mass, $x_{2}$ and the output is the control force, $u$. The dynamic equations of motion of the system under consideration can be written as

$$
\begin{aligned}
& m_{1} s^{2} x_{1}+\left(k_{1}+c_{1} s\right)\left(x_{1}-w\right)+\left(k_{2}+c_{2} s\right)\left(x_{1}-x_{2}\right)=u \\
& m_{2} s^{2} x_{2}+\left(k_{2}+c_{2} s\right)\left(x_{2}-x_{1}\right)=0
\end{aligned}
$$

where $s$ is a Laplace transform variable. From Eq. (8), motion of mass $m_{1}$ can be written as

$$
x_{1}=\frac{u+\left(k_{1}+c_{1} s\right) w+\left(k_{2}+c_{2} s\right) x_{2}}{m_{1} s^{2}+\left(c_{1}+c_{2}\right) s+\left(k_{1}+k_{2}\right)}
$$

Substituting Eq. (10) in (9), we get

$$
x_{2}=G_{1} u+G_{2} w
$$

where

$$
\begin{aligned}
& G_{1}=\frac{k_{2}+c_{2} s}{\left(m_{1} s^{2}+\left(c_{1}+c_{2}\right) s+k_{1}+k_{2}\right)\left(m_{2} s^{2}+c_{2} s+k_{2}\right)-\left(k_{2}+c_{2} s\right)^{2}} \\
& G_{2}=\frac{\left(k_{1}+c_{1} s\right)\left(k_{2}+c_{2} s\right)}{\left(m_{1} s^{2}+\left(c_{1}+c_{2}\right) s+k_{1}+k_{2}\right)\left(m_{2} s^{2}+c_{2} s+k_{2}\right)-\left(k_{2}+c_{2} s\right)^{2}}
\end{aligned}
$$

Similarly, the motion of mass $m_{1}$ can be expressed as

$$
x_{1}=G_{3} u+G_{4} w
$$

where transfer functions $G_{3}$ and $G_{4}$ can be obtained by substituting Eq. (11) in (10).

The transfer function from $u$ to $y\left(G_{u}\right)$ in Fig. 2 is equal to

$$
G_{u}=\frac{k_{2}+c_{2} s}{\left(m_{1} s^{2}+\left(c_{1}+c_{2}\right) s+k_{1}+k_{2}\right)\left(m_{2} s^{2}+c_{2} s+k_{2}\right)-\left(k_{2}+c_{2} s\right)^{2}}
$$

The system to be isolated has a mass of $20 \mathrm{~kg}$. The natural frequency and damping ratio are $2 \mathrm{~Hz}$ and $1 \%$, respectively. The mass, natural frequency and damping ratio corresponding to the sensor dynamics are taken as $0.5 \mathrm{~kg}, 500 \mathrm{~Hz}$ and $1 \%$, respectively. The merging frequency $\left(\omega_{f}\right)$ of the filters is set as $50 \mathrm{~Hz}$. The filters are chosen to be complementary to avoid zeros that might creep in due to merging [22]. Fifth order complementary filters are used in the present study whose transfer functions are given by 


$$
\begin{aligned}
& H_{L}=\frac{1.2865 \times 10^{-16}\left(s+3.54 \times 10^{7}\right)(s+26.24)(s+6.541)\left(s^{2}-3.54 \times 10^{7} s+1.253 \times 10^{15}\right)}{(s+622)(s+62.83)(s+6.347)\left(s^{2}+62.83 s+3948\right)} \\
& H_{H}=\frac{s^{3}(s+603.5)(s+150.4)}{(s+622)(s+62.83)(s+6.347)\left(s^{2}+62.83 s+3948\right)}
\end{aligned}
$$

The frequency responses of the filters are shown in Fig. 4. A lead-lag compensator is used as controller, $K$. A lead compensator provides phase lead at higher frequencies. It shifts the root locus of the system to the left half plane and thus improves the stability and responsiveness of the system. A lag compensator introduces phase lag at the lower frequencies and reduces the steady-state error. Lag ensures sufficient phase margins at the lower crossover frequency while the lead does so at the higher crossover frequency. A lead-lag compensator cascades a lead compensator with a lag compensator. It combines the advantages of both the compensators. A typical transfer function of a lead-lag compensator is given by

$$
K(s)=\frac{\left(s-z_{1}\right)\left(s-z_{2}\right)}{\left(s-p_{1}\right)\left(s-p_{2}\right)}
$$

where $p_{1}$ and $z_{1}$ are the pole and zero of the lead compensator while $p_{2}$ and $z_{2}$ are the pole and zero of the lag compensator. They typically follow the relation $\left|p_{1}\right|>\left|z_{1}\right|>\left|z_{2}\right|>\left|p_{2}\right|$. The transfer function of the controller used in the present study is

$$
K=\frac{1.5791 \times 10^{5}(s+23.84)(s+2)}{(s+238.4)(s+0.2)}
$$

The virtual sensor is chosen in such a way that its response near the merging frequency has the same gain and same slope as the real plant. This avoids the undesirable zeros that might appear due to merging. It is assumed that we have a good model of the plant near the merging frequency. One of the simplest choices for virtual sensor $\left(G_{v}\right)$ is the model of the system to be isolated. In the current example, $G_{v}$ is assumed to be the reduced order model of the system (obtained by neglecting the sensor dynamics) to be isolated. That is,

$$
G_{v}=\frac{1}{m_{1} s^{2}+c_{1} s+k_{1}}
$$

The comparison of the frequency response of the plant before fusion, $G_{u}$, and the plant after fusion $\left(G_{v} H_{H}+G_{u} H_{L}\right)$ is shown Fig. 5. It can be seen that there is no phase degradation due to sensor dynamics in the plant after fusion. The comparison of the transfer functions from inertial control, virtual sensor control and their fusion to $x_{2}$ is shown in Fig. 6 . It reas-

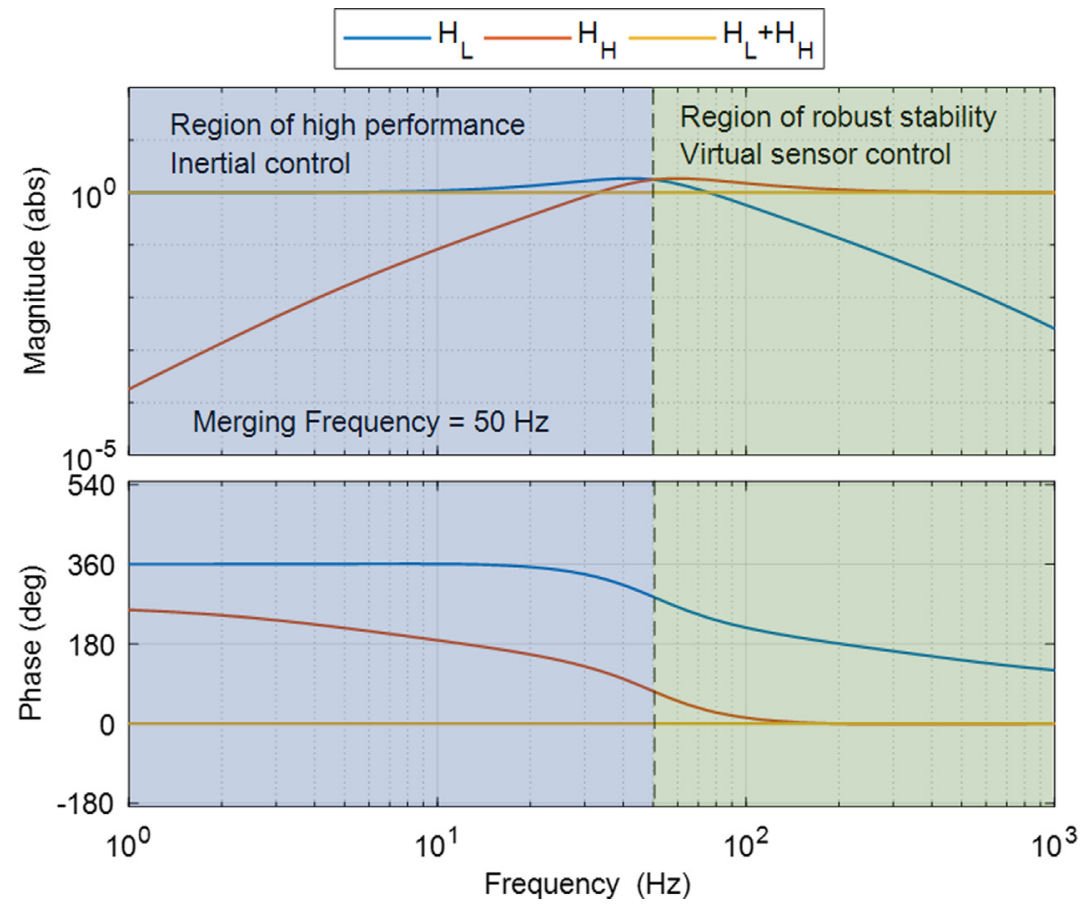

Fig. 4. Frequency response of complementary filters. 

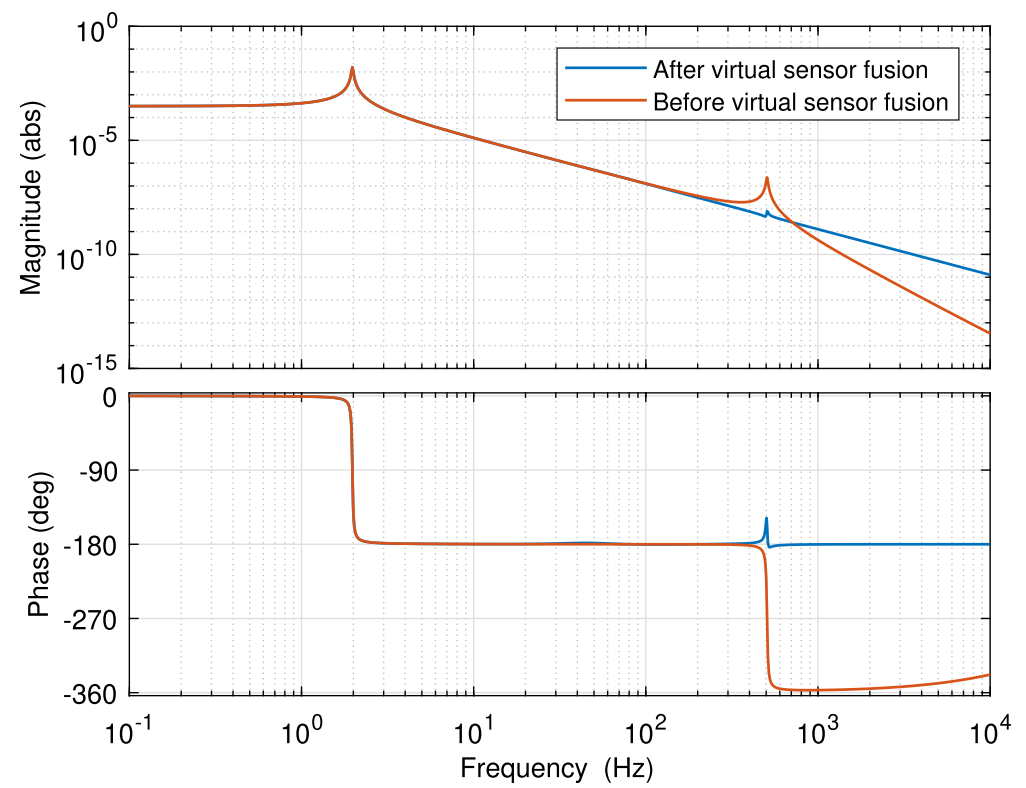

Fig. 5. Frequency response of the plant before fusion $\left(G_{u}\right)$ and the plant after fusion $\left(G_{v} H_{H}+G_{u} H_{L}\right)$.

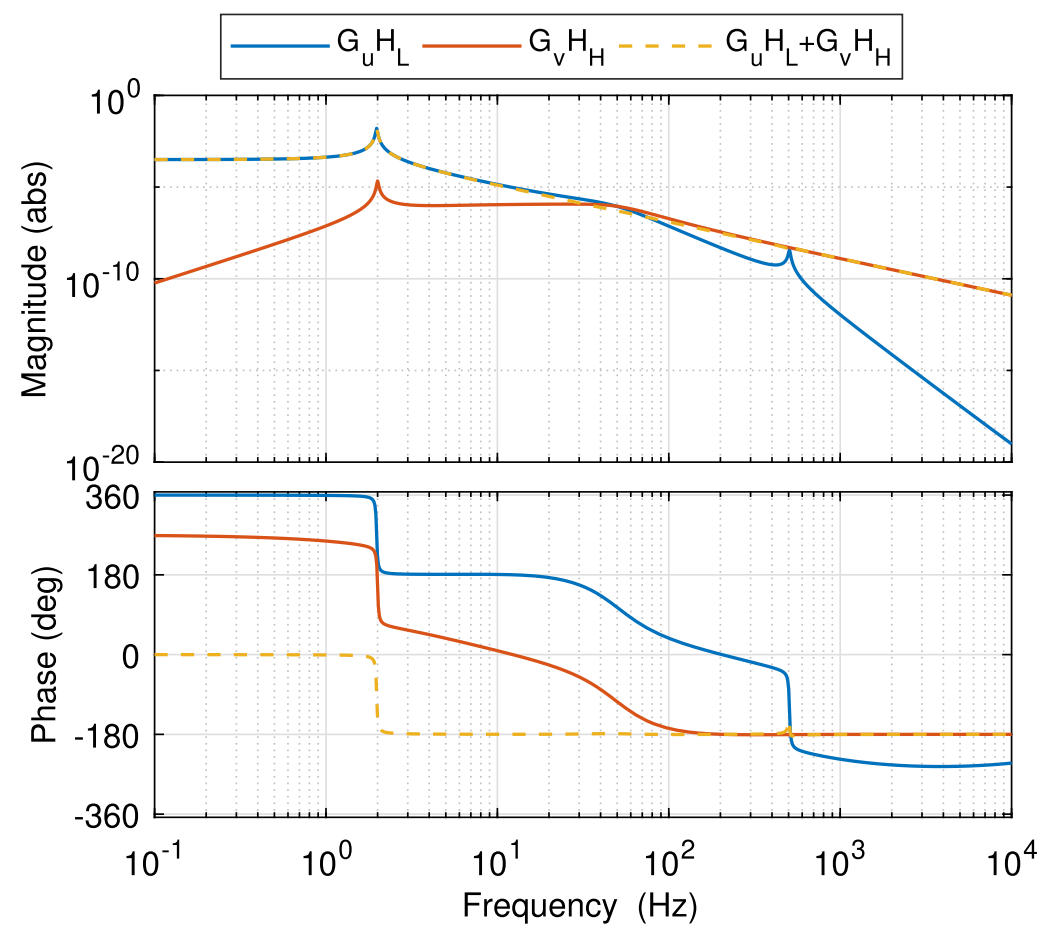

Fig. 6. Comparison of the transfer function $G_{u} H_{L}, G_{v} H_{H}$ and $G_{u} H_{L}+G_{v} H_{H}$.

sures the fact that inertial control is dominant at low frequency while virtual sensor control becomes dominant at high frequency in virtual sensor fusion. The transmissibilties (i.e., the magnitude of the transfer function from $w$ to $x_{1}$ ) of the open and closed loop systems are compared in Fig. 7. The virtual sensor fusion provides good isolation performance below $10 \mathrm{~Hz}$. 




Fig. 7. Comparison of transmissibility for the open and closed loop system.

\section{Robustness to real plant uncertainty}

In this section we demonstrate the robust stability of virtual sensor fusion for the same system studied in the previous section. To this purpose, we will represent the real plant $\left(G_{u}\right)$ as a perturbation of the virtual plant $\left(G_{v}\right)$. In other words, the high frequency peak of $G_{u}$ can be seen as spurious resonance introduced in the system due to sensor dynamics. This is treated as the unmodeled dynamics. To account for unmodeled dynamics (for example, phase lag and high frequency resonances), the plant $\left(G_{u}\right)$ can be represented mathematically using multiplicative uncertainty model [35] given by

$$
\tilde{G}_{u}=G_{v}(1+\Delta(s) W(s))
$$

where, $\tilde{G}_{u}$ is a set of perturbed models of the plant; $\Delta$ is a variable and stable transfer function satisfying $\|\Delta\|_{\infty} \leqslant 1$; and $W$ is a stable weighting transfer function.

The weighting function, $W$, is selected such that it covers all the unmodeled dynamics of the plant. The modeling errors are usually negligible at low frequency. These errors tend to increase with the frequency. Therefore, the obvious choice of the weighting function is a high pass filter. In the present case, the real plant has a spurious resonance at about $500 \mathrm{~Hz}$ with the peak magnitude less than $35 \mathrm{~dB}$. The cutoff frequency of the weighting functions is set to $400 \mathrm{~Hz}$. The magnitude of the weighting transfer function is adjusted to cover all the unmodeled dynamics. The weighting transfer function, $W(s)$, is

$$
W(s)=\frac{70 s^{2}}{(s+2 \pi \cdot 400)^{2}}
$$

The magnitude of the frequency response of real, nominal and perturbed plants are compared in Fig. 8. It can be seen that the perturbed plants cover the spurious resonance of the real plant. The nominal loop gain $\left(L_{n}\right)$ of the plant can be obtained by substituting $G_{u}=G_{v}$ in $L$ (defined in Eq. (1)). That is,

$$
L_{n}=K\left(G_{v} H_{L}+G_{v} H_{H}\right)=K G_{v}\left(H_{L}+H_{H}\right)=K G_{v}
$$

The loop gain of the perturbed models of the plant $\left(L_{p}\right)$ can be written as

$$
\begin{aligned}
L_{p} & =K\left(\tilde{G}_{u} H_{L}+G_{v} H_{H}\right)=K\left(G_{v}(1+\Delta(s) W(s)) H_{L}+G_{v} H_{H}\right)=K\left(G_{v} H_{L}+G_{v} H_{H}+G_{v} \Delta(s) W(s) H_{L}\right) \\
& =L_{n}+K G_{v} \Delta(s) W(s) H_{L}
\end{aligned}
$$

The robust stability analysis can be represented graphically in the complex plane. The graphical representation of the robust stability analysis for virtual sensor fusion is shown in Fig. 9. The blue line in the figure corresponds to the Nyquist plot of the nominal loop gain $\left(L_{n}\right)$. Each point on the blue curve corresponds to the complex frequency response of $L_{n}$ at a particular frequency $(s=j \omega)$. The critical point in the Nyquist plot is -1 , as the closed loop system becomes unstable when 


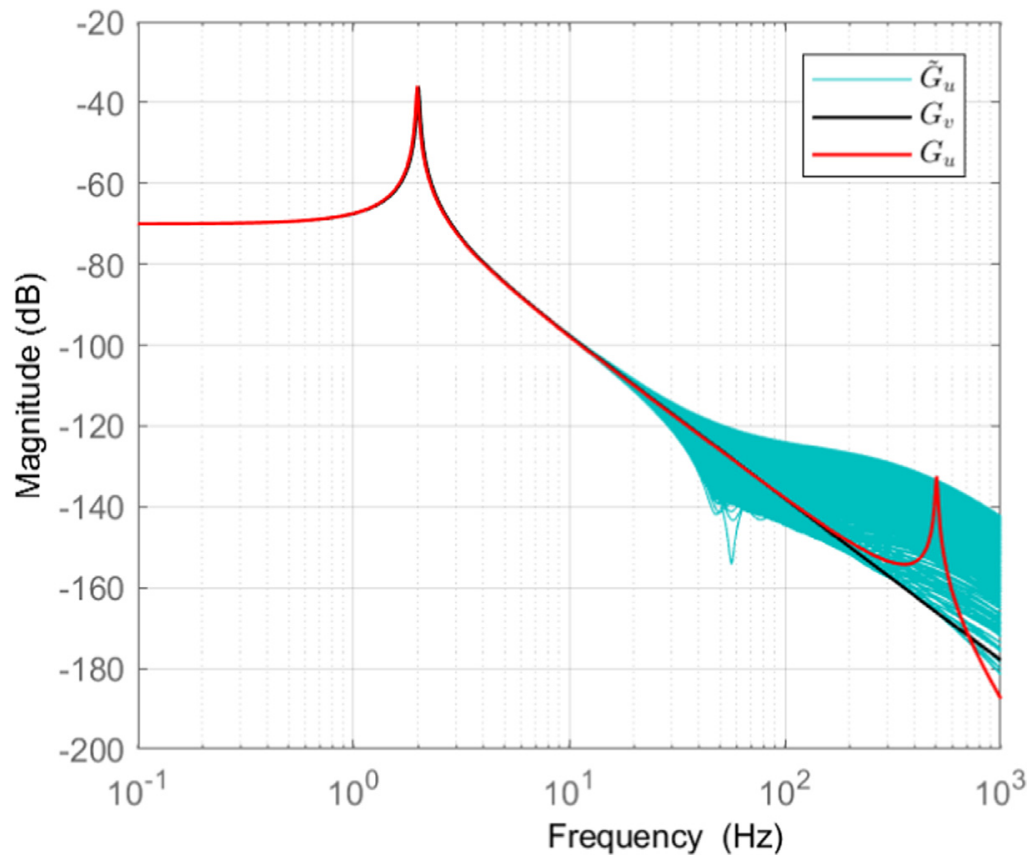

Fig. 8. Bode magnitude plot of nominal plant $\left(G_{v}\right)$, real plant $\left(G_{u}\right)$ with spurious resonance and the perturbed plants $\left(\tilde{G}_{u}\right)$.

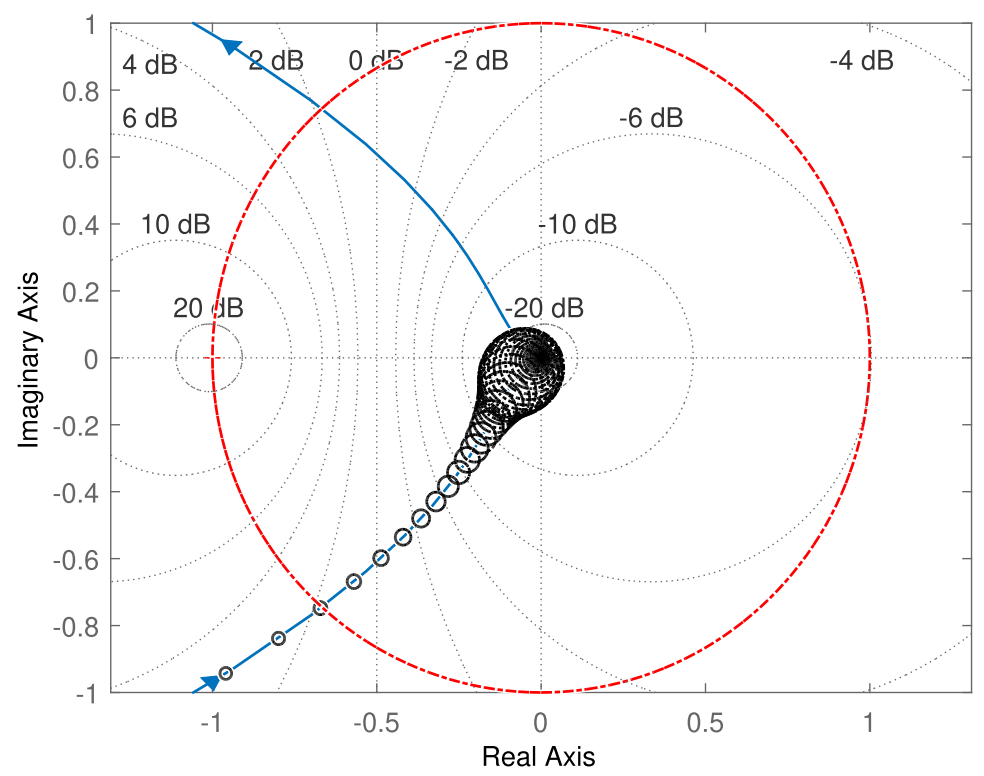

Fig. 9. Graphical representation of the robust stability analysis for virtual sensor fusion.

loop gain is equal -1 . The point closest to the critical point determines the gain and phase margins of the system. Hence, the red circle with the unity radius defines the stability limit. The magnitude of uncertainty, $U_{r}$, in the loop gain of the perturbed model is frequency dependent. Based on Eq. (21), it can be written as

$$
U_{r}=\left|K G_{v} W(s) H_{L}\right|
$$


The points satisfying this magnitude criteria transforms to a circle in the complex plane. This is represented by the black dotted circles. Each circle corresponds to the uncertainty disk at a particular frequency whose radius is equal to $U_{r}$ [36]. The stability margin is defined as the minimum angle with respect to the real axis at which uncertainty disk intersects the unit radius circle centered at origin on the Nyquist plot. It can be seen that even in the presence of uncertainties, the stability margins of the system are not much affected in virtual sensor fusion. To summarize, the algorithm for virtual sensor fusion is given in Algorithm 1.

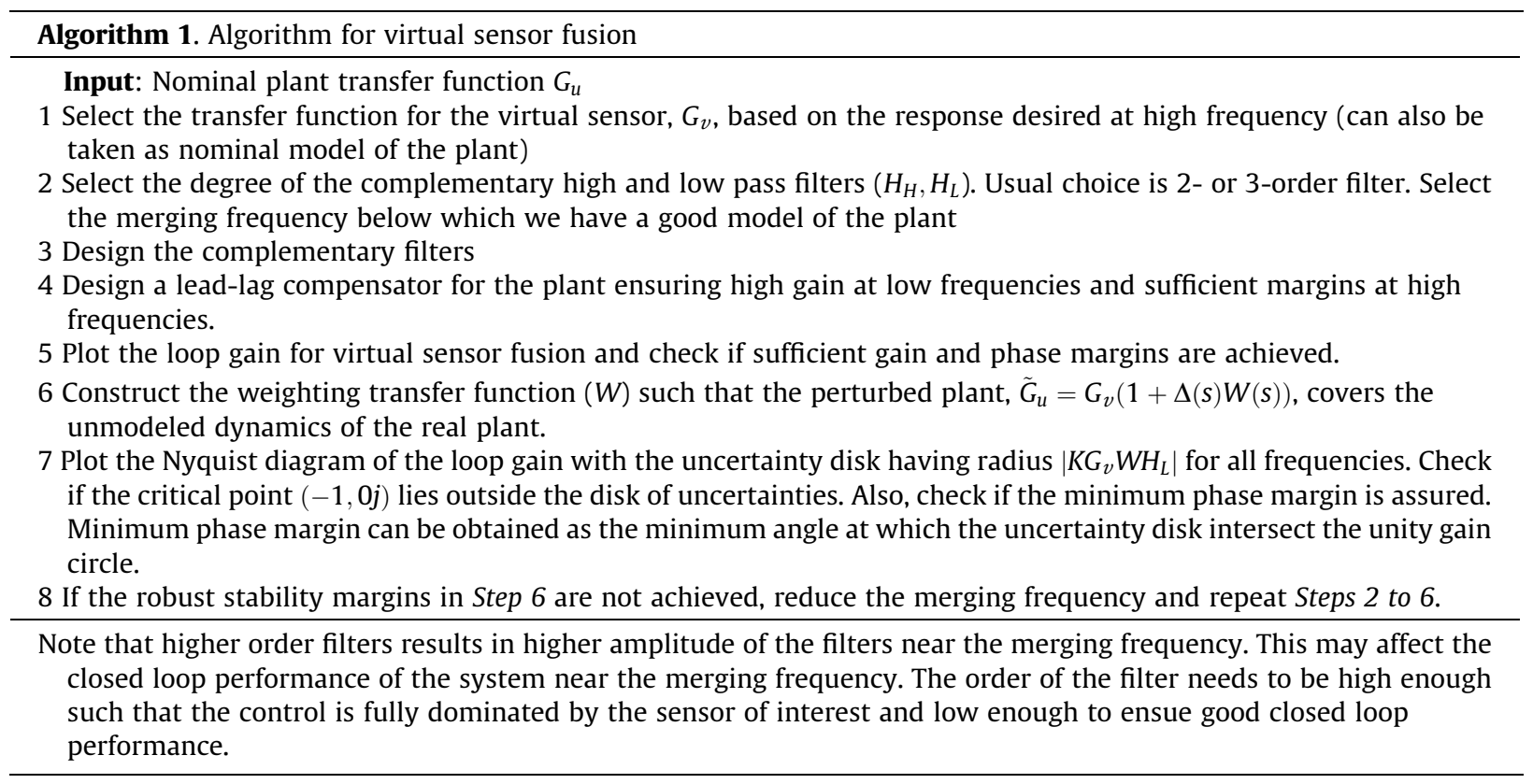

\section{Experimental validation}

This section presents an experimental implementation of the virtual sensor fusion technique. The aim of the experiment is to actively isolate a pendulum from the ground motion.

\subsection{Test setup}

The experiment using virtual sensor fusion was carried out on a pendulum system whose different parts are shown in Fig. 10. It represents a simple lever system with beam acting as a lever (part (2)) and a wedge as fulcrum (part (4)). The masses of the beam and inertial sensor are supported by a spring (part (3)). The motion of the host structure (i.e, the tip of the beam, on the other side of the spring) was isolated from the ground motion using virtual sensor fusion. A voice coil actuator was used to control the host structure motion (part (6)). A customized high-resolution interferometric inertial sensor [37,38], developed in-house, was used for measuring inertial motion of the host structure (part (1)). The inertial ground motion was also measured using a similar sensor. The sensor on the pendulum had two sensing units - loop sensor used for feedback and monitor sensor used for monitoring purpose. The interferometer provided two quadrature signals. The inertial motion is related to the phases of those quadrature signals. The resolution of the interferometric sensor used was $10^{-12} \mathrm{~m} / \sqrt{\mathrm{Hz}}$ at $1 \mathrm{~Hz}$. The controller was implemented in real-time using dSpace MicroLabBox, which was also used for data acquisition (part (5)). The signals were measured with a sampling frequency of $1 \mathrm{kHz}$. The duration of the measurement is set to $2000 \mathrm{~s}$. The transmissibilities are calculated as the quotient between the cross-power spectral density of the input and the output; and the self-power spectral destiny of the input. A 200 s hanning window is applied on the measured signals when calculating the cross-power spectral density and the self-power spectral density.

\subsection{Control strategy}

The control objective of the experiments is to have good isolation of the tip of the beam from the base excitation at low frequency. The system has spurious resonances at high frequencies, against which the stability of the system needs to be ensured. A good model of the plant is available up to $7 \mathrm{~Hz}$. In the experiments, first order complementary filters were used for virtual sensor fusion (higher order filters were not realized at the time of the experiments). The high/low frequency roll- 


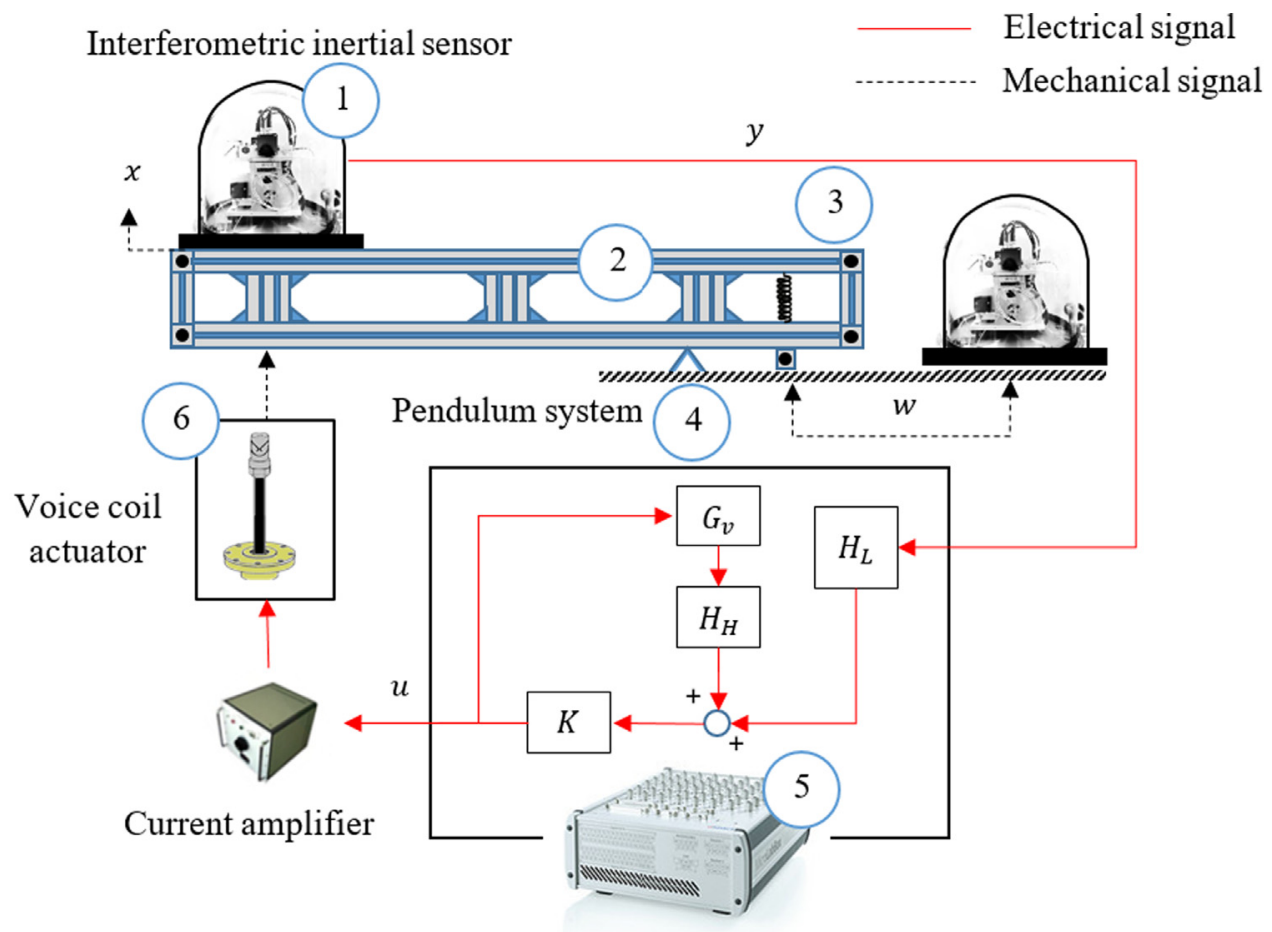

Digital controller

(a)
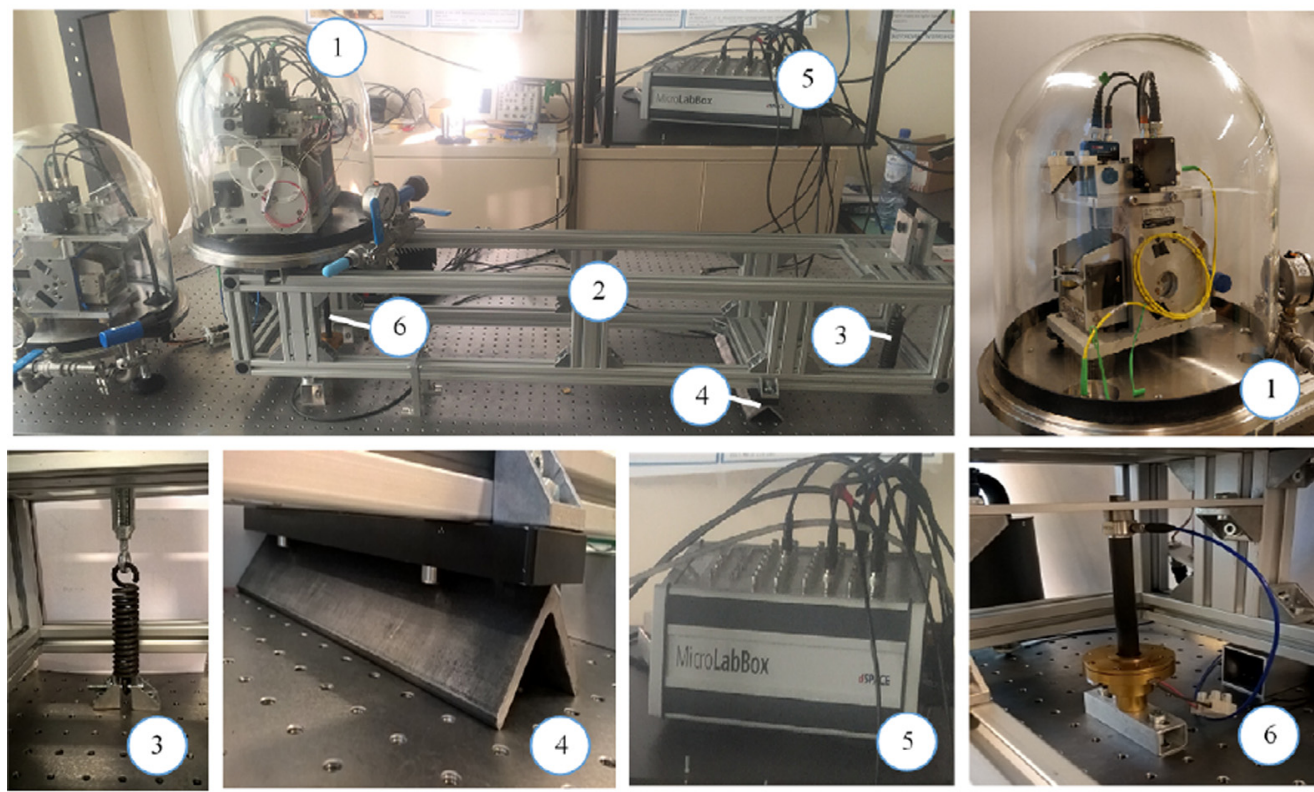

(b)

Fig. 10. Experimental setup - (a) schematic diagram and (b) actual setup.

off of a high-/low-pass filter depends on the order of the filter. This implies that a higher order filter will have more attenuation per decade compared to a lower order filter. The use of first order filter was not having enough attenuation and the spurious resonances were present in the open loop gain of the virtual sensor fusion near the merging frequency. These spurious resonance near the merging frequencies were attenuated using notch filters. Hence, in order to use first order filter, one needs to have good knowledge of the plant near the merging frequency to achieve the desired level of attenuation. The merg- 

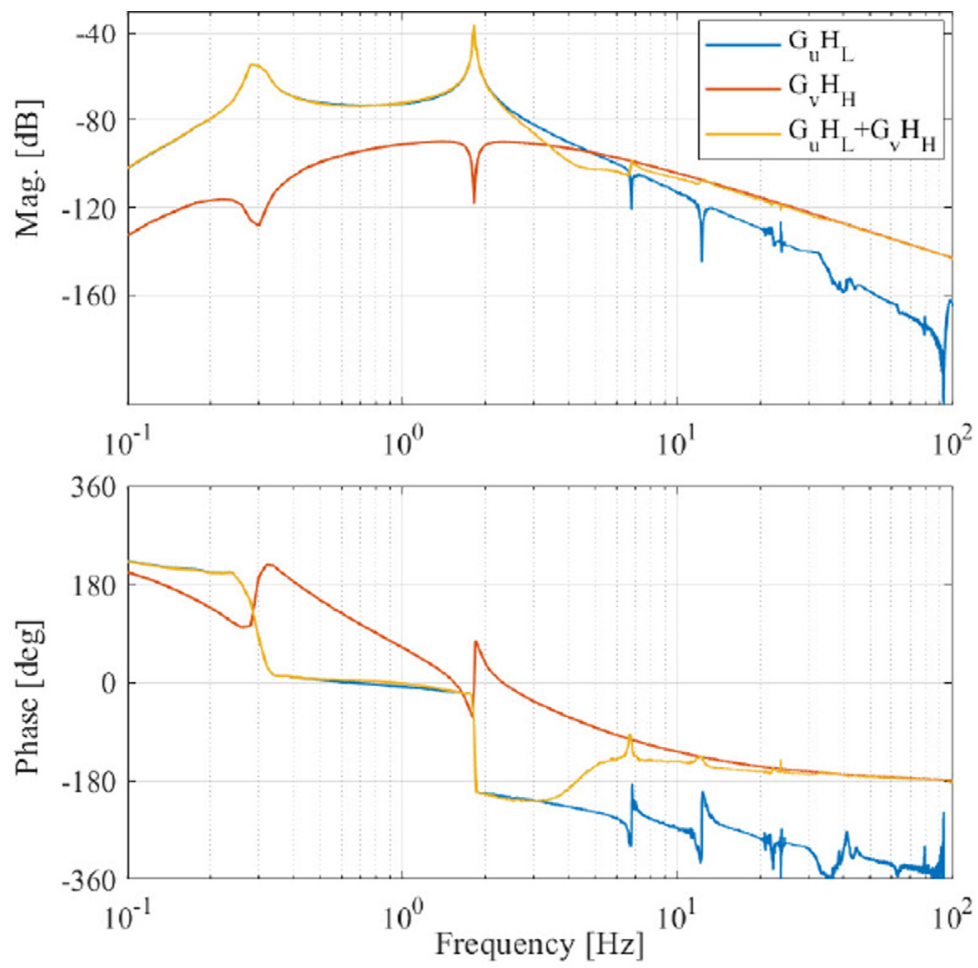

Fig. 11. Comparison of the transfer functions from inertial control, virtual sensor control and their fusion to host structure motion.

ing frequency for the virtual sensor fusion was taken as $5 \mathrm{~Hz}$. Appropriate notch filters were augmented with the complementary filters in the vicinity of the merging frequency. We would like to highlight that these modifications to the complementary filters would not be required if higher order filters are used, which provides the desired level of attenuation and also prevents the spurious resonances from appearing in the open loop frequency response. Reduced order model of the plant, capturing dynamics up to $7 \mathrm{~Hz}$ followed by roll-off, was used as the virtual sensor. A lead-lag compensator was used for the design of controller, $K$. The transfer functions from inertial control times low pass filter $\left(G_{u} H_{L}\right)$, virtual sensor control

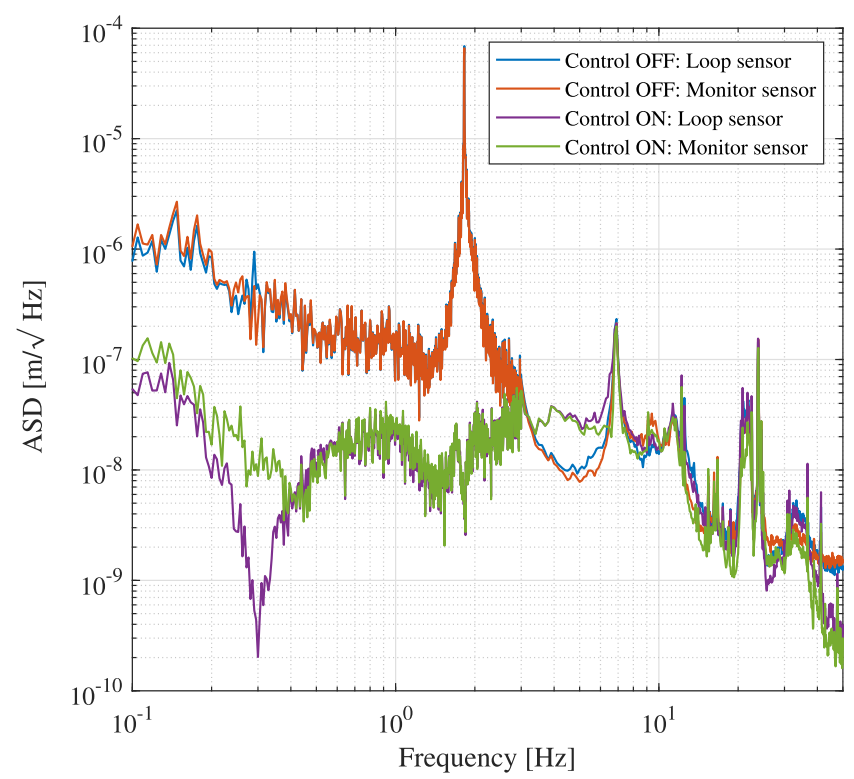

Fig. 12. ASD of the host structure motion measured using interferometric inertial sensor with and without active control. 

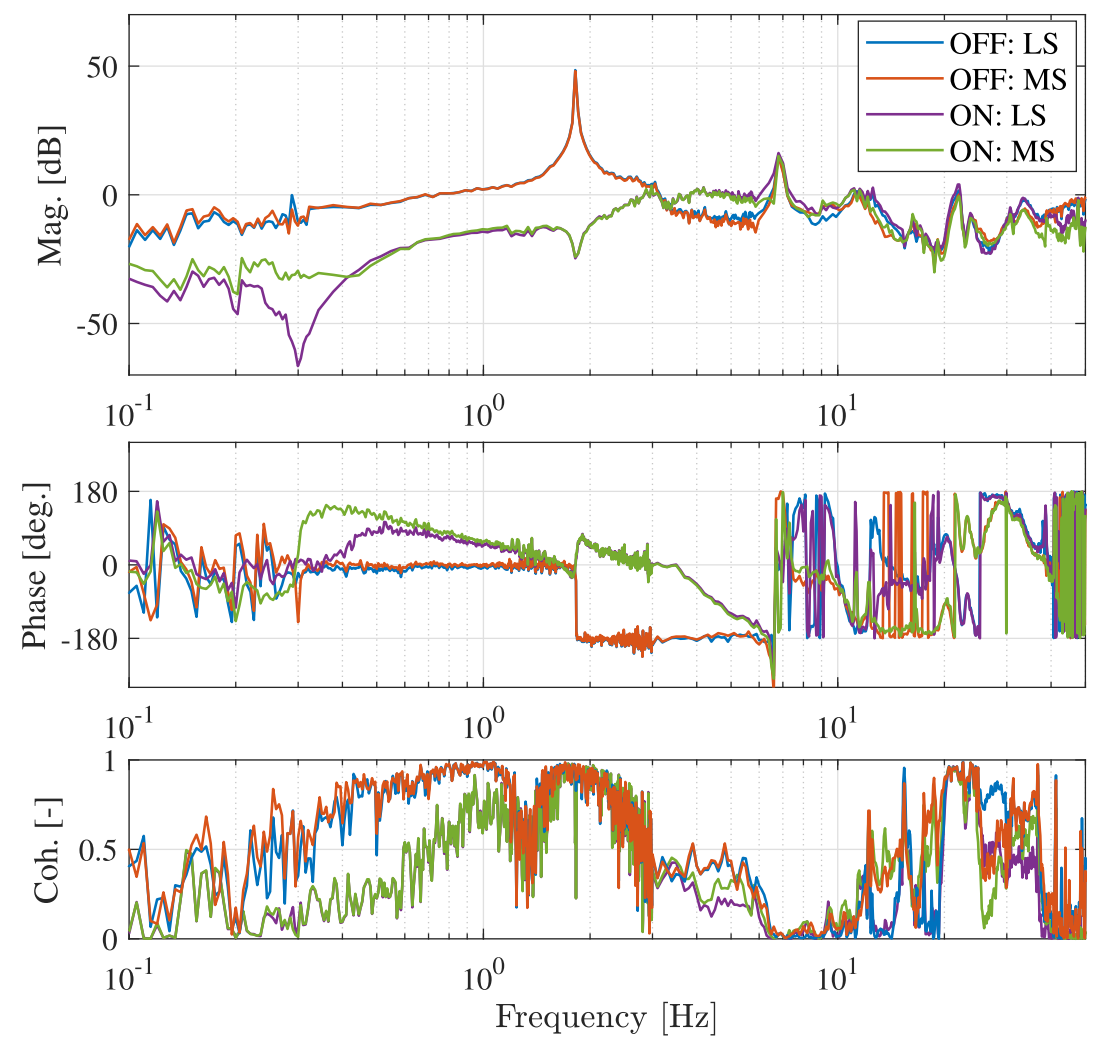

Fig. 13. Comparison of the transfer function from ambient ground motion to the host structure motion with and without active control (MS: Monitor sensor, LS: Loop sensor).

times highpass filter $\left(G_{v} H_{H}\right)$ and virtual sensor fusion $\left(G_{u} H_{L}+G_{v} H_{H}\right.$, i.e, the sum of low frequency inertial control and high frequency virtual sensor control) to the host structure motion are compared in Fig. 11. A lot of fluctuations are observed in the magnitude and phase response of the inertial control $\left(G_{u} H_{L}\right)$ above $20 \mathrm{~Hz}$ which can affect the stability of the system at high frequency. On the other hand, the virtual sensor fusion control $\left(G_{u} H_{L}+G_{v} H_{H}\right)$ is found to be smooth and devoid of any such fluctuations at high frequency. Thus, ensuring the robustness of the system at high frequency.

\subsection{Results}

The amplitude spectral densities (ASDs) of the host structure as recorded by the interferometric sensor with and without control are shown in Fig. 12. It is observed that the ASD measured with the loop sensor is lower than that measured with the monitor sensor. The differences in the loop and monitor sensor readings are attributed to two factors. First, the two sensors have difference noise floors. The second reason being the mismatch in the resonance frequency of the two sensors. Around $0.3 \mathrm{~Hz}$, the extra reductions are not observed in the monitor sensor. This is due to fact that its resonance frequency is slightly different than that of the in-loop sensor. In other words, the sensitivity of the monitor sensor is not as good as that of the inloop sensor at $0.3 \mathrm{~Hz}$. The noise of the monitor sensor dominates its output when the controller is activated and the actual motion of the frame is not precisely measured by the monitor sensor around this frequency. The noise of the loop sensor drives the actuator which in turn forces the host structure to follow the noise. The virtual sensor fusion is able to attenuate the motion of the host structure by ten times around $0.1 \mathrm{~Hz}$. This demonstrates the effectiveness of the proposed strategy. The transmissibilities between the host structure and ground motions with and without control are compared in Fig. 13. The magnitude of control ON transfer function is higher than the control OFF transfer functions near the merging frequency. In the vicinity of the merging frequency, there is increase in the controller gain as evident from the bumps in the filters frequency response near merging frequencies in Fig. 4. Nevertheless, good isolation performance is observed for the frequencies less than $3 \mathrm{~Hz}$.

\section{Concluding remarks}

A new technique, termed as "virtual sensor fusion", has been presented in which the signal from real and virtual sensors are fused in synergy to achieve good performance at lower frequencies while ensuring the stability of the system at high 
frequencies. The real sensor is chosen as inertial sensor while the virtual is simply a stable transfer function exhibiting desired high frequency response. The real and virtual sensors are augmented with high and low pass complementary filters such that the control at low frequency is dominated by the inertial sensor and at high frequency by the virtual sensor. The merging frequency of the two filters is usually taken as the frequency below which the dynamics of the real plant are well represented by its model. Since the control action at high frequency is dominated by the virtual sensor, the effect of spurious resonances that might exist at high frequency (unmodeled dynamics) does not appear in the feedback loop. Hence, the stability and robustness of the system is ensured in the high frequency region. The effectiveness of the proposed control strategy is demonstrated by applying it to the active seismic vibration isolation system. A robustness analysis is also carried out by representing unmodeled dynamics as multiplicative uncertainties and evaluating guaranteed stability margins. Finally, the experimental validation of virtual sensor fusion is carried out on a pendulum system. Virtual sensor fusion is found to reduce the transmissibilty by an order of 10 at lower frequencies while keeping the system robustly stable. The future work will focus on the design of higher order complementary filters through optimization to achieve desired trade-off between performance and robustness. This technique can be further generalized to multi-input multi-output (MIMO) systems using specially designed complementary filters.

\section{CRediT authorship contribution statement}

Mohit Verma: Methodology, Investigation, Software, Writing - original draft. Thomas Dehaeze: Conceptualization, Writing - review \& editing. Guoying Zhao: Data curation, Writing - review \& editing. Jennifer Watchi: Investigation, Validation, Writing - review \& editing. Christophe Collette: Funding acquisition, Conceptualization, Supervision, Writing - review \& editing.

\section{Declaration of Competing Interest}

The authors declare that they have no known competing financial interests or personal relationships that could have appeared to influence the work reported in this paper.

\section{Acknowledgements}

The authors gratefully acknowledge the French Community for financing FRIA grant of Jennifer Watchi (Grant No. FC 27289) and Thomas Dehaeze (Grant No. FC 31597). Authors also acknowledge FRS-FNRS for funding this research (grant agreement F.4536.17). The authors would like also to thank the Klaus Stammler and Edgar Wetzig from the Federal Institute for Geosciences and Natural Resources in Hannover and Michel Can Camp from the Royal Observatory of Belgium for lending STS-1V seismometers for the tests. The authors also thank anonymous reviewers whose feedback helped to improve the quality of the manuscript.

\section{References}

[1] X. Li, Simultaneous, fault-tolerant vibration isolation and pointing control of flexure jointed hexapods, Ph.D. thesis, University of Wyoming, 2000.

[2] M. Verma, V. Lafarga, M. Baron, C. Collette, Active stabilization of unmanned aerial vehicle imaging platform, Journal of Vibration and Control (2020), 1077546320905494.

[3] M. Verma, C. Collette, Active vibration isolation system for drone cameras, in: Proceedings of International Conference on Vibration Problems: ICOVP 2019, 2019.

[4] T. Tang, S.X. Niu, T. Yang, B. Qi, Q.L. Bao, Vibration rejection of tip-tilt mirror using improved repetitive control, Mechanical Systems and Signal Processing 116 (2019) 432-442.

[5] C. Collette, K. Artoos, A. Kuzmin, S. Janssens, M. Sylte, M. Guinchard, C. Hauviller, Active quadrupole stabilization for future linear particle colliders, Nuclear Instruments and Methods in Physics Research Section A: Accelerators, Spectrometers, Detectors and Associated Equipment 621 (1-3) (2010) $71-78$.

[6] F. Matichard, B. Lantz, K. Mason, R. Mittleman, B. Abbott, S. Abbott, E. Allwine, S. Barnum, J. Birch, S. Biscans, et al, Advanced ligo two-stage twelve-axis vibration isolation and positioning platform. Part 2: Experimental investigation and tests results, Precision Engineering 40 (2015) $287-297$.

[7] G. Zhao, B. Ding, J. Watchi, A. Deraemaeker, C. Collette, Experimental study on active seismic isolation using interferometric inertial sensors, Mechanical Systems and Signal Processing 145 (2020) 106959.

[8] S. Yi, B. Yang, G. Meng, Microvibration isolation by adaptive feedforward control with asymmetric hysteresis compensation, Mechanical Systems and Signal Processing 114 (2019) 644-657.

[9] L. Vaillon, C. Philippe, Passive and active microvibration control for very high pointing accuracy space systems, Smart Materials and Structures 8 (6) (1999) 719.

[10] M. Verma, A. Pece, S. Hellegouarch, J. Watchi, G. Durand, S. Chesné, C. Collette, Dynamic stabilization of thin aperture light collector space telescope using active rods, Journal of Astronomical Telescopes, Instruments, and Systems 6 (1) (2020) 014002

[11] W.B. Gevarter, Basic relations for control of flexible vehicles, AIAA Journal 8 (4) (1970) 666-672.

[12] M. Verma, V. Lafarga, C. Collette, Perfect collocation using self-sensing electromagnetic actuator: application to vibration control of flexible structures, Sensors and Actuators A: Physical (2020) 112210.

[13] F. Matichard, B. Lantz, K. Mason, R. Mittleman, B. Abbott, S. Abbott, E. Allwine, S. Barnum, J. Birch, S. Biscans, D. Clark, D. Coyne, D. DeBra, R. DeRosa, S. Foley, P. Fritschel, J. Giaime, C. Gray, G. Grabeel, J. Hanson, M. Hillard, J. Kissel, C. Kucharczyk, A. Le Roux, V. Lhuillier, S. Macinnis, B. O’Reilly, D. Ottaway, H. Paris, M. Puma, H. Radkins, C. Ramet, M. Robinson, L. Ruet, P. Sareen, D. Shoemaker, A. Stein, J. Thomas, M. Vargas, J. Warner, Design and performance overview of advanced ligo two-stage vibration isolation and alignment system, ASPE Conference.

[14] J.F. Yocum, L.I. Slafer, Control system design in the presence of severe structural dynamics interactions, Journal of Guidance, Control, and Dynamics 1 (2) (1978) 109-116.

[15] L. Zuo, Element and system design for active and passive vibration isolation, Ph.D. thesis, Massachusetts Institute of Technology, 2004. 
[16] N. Robertson, P. Fritschel, B. Shapiro, C. Torrie, M. Evans, Design of a tuned mass damper for high quality factor suspension modes in advanced ligo, Review of Scientific Instruments 88 (3) (2017) 035117.

[17] W. Ma, Y. Yang, J. Yu, General routine of suppressing single vibration mode by multi-dof tuned mass damper: application of three-dof, Mechanical Systems and Signal Processing 121 (2019) 77-96.

[18] E. Matta, Ball vibration absorbers with radially-increasing rolling friction, Mechanical Systems and Signal Processing 132 (2019) 353-379.

[19] W. Hua, Low frequency vibration isolation and alignment system for advanced ligo, Ph.D. thesis, stanford University, 2005.

[20] D. Tjepkema, J. van Dijk, H. Soemers, Sensor fusion for active vibration isolation in precision equipment, Journal of Sound and Vibration 331 (4) (2012) $735-749$.

[21] G. Losurdo, G. Calamai, E. Cuoco, L. Fabbroni, G. Guidi, M. Mazzoni, R. Stanga, F. Vetrano, L. Holloway, D. Passuello, et al, Inertial control of the mirror suspensions of the virgo interferometer for gravitational wave detection, Review of Scientific Instruments 72 (9) (2001) $3653-3661$.

[22] C. Collette, F. Matichard, Sensor fusion methods for high performance active vibration isolation systems, Journal of Sound and Vibration 342 (2015) $1-$ 21.

[23] Y.-S. Lee, S. Elliott, Active position control of a flexible smart beam using internal model control, Journal of Sound and Vibration 242 (5) (2001) 767791.

[24] R. De Keyser, C. Copot, A. Hernandez, C. Ionescu, Discrete-time internal model control with disturbance and vibration rejection, Journal of Vibration and Control 23 (1) (2017) 3-15.

[25] T.H. Lee, T.S. Low, A. Al-Mamun, C. Tan, Internal model control (imc) approach for designing disk drive servo-controller, IEEE Transactions on Industrial Electronics 42 (3) (1995) 248-256.

[26] S. Endo, H. Kobayashi, C. Kempf, S. Kobayashi, M. Tomizuka, Y. Hori, Robust digital tracking controller design for high-speed positioning systems, Control Engineering Practice 4 (4) (1996) 527-536.

[27] J. Back, H. Shim, Adding robustness to nominal output-feedback controllers for uncertain nonlinear systems: a nonlinear version of disturbance observer, Automatica 44 (10) (2008) 2528-2537.

[28] C. Bohn, A. Cortabarria, V. Härtel, K. Kowalczyk, Active control of engine-induced vibrations in automotive vehicles using disturbance observer gain scheduling, Control Engineering Practice 12 (8) (2004) 1029-1039.

[29] B.A. Guvenc, L. Guvenc, Robust two degree-of-freedom add-on controller design for automatic steering, IEEE Transactions on Control Systems Technology 10 (1) (2002) 137-148.

[30] M.T. White, M. Tomizuka, C. Smith, Improved track following in magnetic disk drives using a disturbance observer, IEEE/ASME Transactions on Mechatronics 5 (1) (2000) 3-11.

[31] M.G. Tehrani, J.E. Mottershead, A.T. Shenton, Y.M. Ram, Robust pole placement in structures by the method of receptances, Mechanical Systems and Signal Processing 25 (1) (2011) 112-122.

[32] X. Xu, J. Liu, S. Chen, Internal model control for reduction of bias and harmonic currents in hybrid magnetic bearing, Mechanical Systems and Signal Processing 115 (2019) 70-81.

[33] Q. Wu, X. Wang, L. Hua, M. Xia, Dynamic analysis and time optimal anti-swing control of double pendulum bridge crane with distributed mass beams, Mechanical Systems and Signal Processing 144 (2020) 106968.

[34] T. Dehaeze, M. Verma, C. Collette, Complementary filters shaping using $H_{\infty}$ synthesis, in: 2019 7th International Conference on Control, Mechatronics and Automation (ICCMA), IEEE, 2019, pp. 459-464.

[35] J.R. Ryoo, T.-Y. Doh, M.J. Chung, Robust disturbance observer for the track-following control system of an optical disk drive, Control Engineering Practice 12 (5) (2004) 577-585.

[36] J.C. Doyle, B.A. Francis, A.R. Tannenbaum, Feedback control theory, Courier Corporation (2013).

[37] B. Ding, G. Zhao, J. Watchi, C. Collette, Huddle test of optical inertial sensors combined with slightly damped mechanics, Proceedings of ISMA2018 and USD2018 (2018) 1471-1478.

[38] B. Ding, J. Watchi, C. Collette, Development of a high resolution optical inertial sensor, in: Proceedings of 7th World Conference on Structural Control and Monitoring, 7WCSCM, Qingdao, China, 2018, p. Paper No. 263. 